\title{
Construction of Three Quadrature Formulas of Eighth Order and Their Application for Approximating Series
}

\author{
Bogusław Bożek, Wiesław Solak, Zbigniew Szydełko \\ Faculty of Applied Mathematics, AGH University of Science and Technology, Cracow, Poland \\ Email: bozek@agh.edu.pl, solak@agh.edu.pl, szydelko@agh.edu.pl
}

Received 29 April 2015; accepted 6 June 2015; published 9 June 2015

Copyright (C) 2015 by authors and Scientific Research Publishing Inc.

This work is licensed under the Creative Commons Attribution International License (CC BY). http://creativecommons.org/licenses/by/4.0/

(c) () Open Access

\section{Abstract}

In this paper, three types of three-parameters families of quadrature formulas for the Riemann's integral on an interval of the real line $I(f)=\int_{a}^{b} f(x) \mathrm{d} x$ are carefully studied. This research is a continuation of the results in the [1]-[3]. All these quadrature formulas are not based on the integration of an interpolant as so as the Gregory rule, a well-known example in numerical quadrature of a trapezoidal rule with endpoint corrections of a given order (see [4]). In some natural restrictions on the parameters we construct the only one quadrature formula of the eight order which belongs to the first, second and third family. For functions whose 8th derivative is either always positive or always negative, we use these quadrature formulas to get good two-sided bound on $I(f)$. Additionally, we apply these quadratures to obtain the approximate sum of slowly convergent series $s=\sum_{i=0}^{\infty} a_{i}$, where $a_{i} \in \mathbb{R}$.

\section{Keywords}

Quadrature and Cubature Formulas, Numerical Integration

\section{Introduction}

We consider the three-parameters families $\mathcal{S}^{\alpha, \beta, \gamma}, \mathcal{P}^{\alpha, \beta, \gamma}, \mathcal{R}^{\alpha, \beta, \gamma}$ of quadrature formulas for the integral $I(f)=\int_{a}^{b} f(x) \mathrm{d} x$. These quadratures are linear combinations of the quadrature investigated in papers [1]-[3] respectively. The error estimates are calculated in dependence of the parameters $\alpha, \beta, \gamma$ and then in some 
natural restrictions on them these are investigated the quadrature formulas of the 8th order. The desired conclusions are made by means of properties of Peano kernels using substantially well-known error formulas. We construct the only one quadrature formula of the eight order which belongs to the family $\mathcal{S}^{\alpha, \beta, \gamma}$, the only one quadrature formula of the eight order too, which belongs to the family $\mathcal{P}^{\alpha, \beta, \gamma}$ and the only one quadrature formula of the eight order too, which belongs to the family $\mathcal{R}^{\alpha, \beta, \gamma}$. Because of the Peano kernels for these quadratures have different signs, for functions whose 8th derivative is either always positive or always negative we use these quadrature formulas to get good bounds on $I(f)$. So, by suitable choice of parameters one can increase quadrature order from two or four respectively to eight.

\section{The Three-Parameters Family of Quadrature Formulas $\mathcal{S}^{\alpha, \beta, \gamma}$}

We consider family of quadrature formulas $\mathcal{S}^{\alpha, \beta, \gamma}$ given by

$$
\begin{aligned}
& \mathcal{S}^{\alpha, \beta, \gamma}(f)= \frac{h}{2} \gamma \sum_{j=1}^{n}\left(f\left(a+\frac{2 j-1+\alpha}{2} h\right)+f\left(a+\frac{2 j-1-\alpha}{2} h\right)\right) \\
&+\frac{h}{2}(1-\gamma) \sum_{j=1}^{n}\left(f\left(a+\frac{2 j-1+\beta}{2} h\right)+f\left(a+\frac{2 j-1-\beta}{2} h\right)\right) \\
& \alpha, \beta \in[0,1], \quad \gamma \in[0,1], \quad h=\frac{b-a}{n}, \quad n \in \mathbb{N}
\end{aligned}
$$

for integral $I(f)=\int_{a}^{b} f(x) \mathrm{d} x$. This family generalizes the family $Q^{\delta}$ discussed in [1], here it is enough to put $\alpha=\beta, \gamma=\frac{1}{2}, \delta=\frac{\alpha+1}{2}$.

For arbitrary $\alpha, \beta, \gamma$ the quadrature formula $\mathcal{S}^{\alpha, \beta, \gamma}$ is of the second order. The error $E^{\alpha, \beta, \gamma}(f)=I(f)-\mathcal{S}^{\alpha, \beta, \gamma}(f)$ for the polynomials $t, t^{2}, \cdots$ is equal

$$
\begin{aligned}
& E^{\alpha, \beta, \gamma}(t)=0, \\
& E^{\alpha, \beta, \gamma}\left(t^{2}\right)=\frac{(b-a)^{3}}{12 n^{2}}\left(1+3 \beta^{2}(\gamma-1)-3 \alpha^{2} \gamma\right), \\
& E^{\alpha, \beta, \gamma}\left(t^{3}\right)=\frac{(b-a)^{3}(a+b)}{8 n^{2}}\left(1+3 \beta^{2}(\gamma-1)-3 \alpha^{2} \gamma\right) .
\end{aligned}
$$

If a triple $(\alpha, \beta, \gamma)$ is a root of the polynomial $\psi_{1}(\alpha, \beta, \gamma)=1+3 \beta^{2}(\gamma-1)-3 \alpha^{2} \gamma$ the range of quadrature formula increases. These triples we can write in the form $(\alpha(\beta, \gamma), \beta, \gamma)$ with

$$
\alpha(\beta, \gamma)=\sqrt{\frac{1+3(\gamma-1) \beta^{2}}{3 \gamma}}
$$

where $(\beta, \gamma) \in D_{01}:=\left\{(\beta, \gamma) \in[0,1]^{2}:(1-\gamma) \beta^{2}<\frac{1}{3}\right\}$. Then every $\mathcal{S}^{\alpha(\beta, \gamma), \beta, \gamma}$ is of the fourth order, and moreover

$$
\begin{aligned}
& E^{\alpha(\beta, \gamma), \beta, \gamma}\left(t^{i}\right)=0 \quad \text { for } i=1,2,3, \\
& E^{\alpha(\beta, \gamma), \beta, \gamma}\left(t^{4}\right)=\frac{(b-a)^{5}}{720 n^{4}} \frac{1}{\gamma}\left(15 \beta^{2}\left(3 \beta^{2}-2\right)(\gamma-1)+9 \gamma-5\right), \\
& E^{\alpha(\beta, \gamma), \beta, \gamma}\left(t^{5}\right)=\frac{(b-a)^{5}(a+b)}{288 n^{4}} \frac{1}{\gamma}\left(15 \beta^{2}\left(3 \beta^{2}-2\right)(\gamma-1)+9 \gamma-5\right) .
\end{aligned}
$$

If the pair $(\beta, \gamma)$ is a root of the polynomial $\psi_{2}(\beta, \gamma)=15 \beta^{2}\left(3 \beta^{2}-2\right)(\gamma-1)+9 \gamma-5$ then the range of 
quadrature increases as before. We can write these pairs in the form $(\beta(\gamma), \gamma)$ where

$$
\beta(\gamma):=\frac{1}{\sqrt{15}} \sqrt{\frac{5 \gamma-5+2 \sqrt{5} \sqrt{\gamma-\gamma^{2}}}{\gamma-1}}
$$

for $\gamma \in\left[0, \frac{5}{9}\right]$.

Every quadrature $\mathcal{S}^{\alpha(\beta(\gamma), \gamma), \beta(\gamma), \gamma}$ is of the six order but we must restrict the interval for $\gamma$. The quadrature nodes belongs to interval $[a, b]$ only for $\gamma \in\left[\frac{1}{6}, \frac{5}{9}\right]$. Graphs of the functions

$$
\alpha(\beta(\gamma), \gamma)=\frac{1}{\sqrt{15}} \sqrt{5+2 \sqrt{\frac{5-5 \gamma}{\gamma}}}
$$

and $\beta(\gamma)$ are presented on the Figure 1 .

In this case we have

$$
\begin{aligned}
& E^{\alpha(\beta(\gamma), \gamma), \beta(\gamma), \gamma}\left(t^{i}\right)=0 \quad \text { for } i=1, \cdots, 5 \\
& E^{\alpha(\beta(\gamma), \gamma), \beta(\gamma), \gamma}\left(t^{6}\right)=-\frac{(b-a)^{7}}{37800 n^{6}} \frac{1}{(\gamma-1) \sqrt{\gamma}}\left((14 \gamma-7) \sqrt{5-5 \gamma}+10 \sqrt{\gamma}-10 \gamma^{\frac{3}{2}}\right), \\
& E^{\alpha(\beta(\gamma), \gamma), \beta(\gamma), \gamma}\left(t^{7}\right)=-\frac{(b-a)^{7}(a+b)}{10800 n^{6}} \frac{1}{(\gamma-1) \sqrt{\gamma}}\left((14 \gamma-7) \sqrt{5-5 \gamma}+10 \sqrt{\gamma}-10 \gamma^{\frac{3}{2}}\right) .
\end{aligned}
$$

The six order Peano kernel $K_{6}^{\mathcal{S}}(\gamma, x):=\frac{1}{5 !} \int_{x}^{b}(t-x)^{5} \mathrm{~d} t-\mathcal{S}^{\alpha(\beta(\gamma), \gamma), \beta(\gamma), \gamma}(p(t))$ where $p(t)=\frac{(t-x)_{+}^{5}}{5 !}$. This kernel is a periodic function with period $h$ and on every interval $[a+i h, a+(i+1) h]$ is symmetrical respect to its midpoint. So, it is enough to define it on the interval $\left[b-\frac{h}{2}, b\right]$ :

$$
K_{6}^{\mathcal{S}}(\gamma, x)=\left\{\begin{aligned}
\frac{(b-x)^{6}}{6 !}-\frac{h}{2 \times 5 !}\left(\gamma\left(a+\frac{2 n-1+\alpha(\beta(\gamma), \gamma)}{2} h-x\right)^{5}+(1-\gamma)\left(a+\frac{2 n-1+\beta(\gamma)}{2} h-x\right)^{5}\right) & \\
& \text { for } x \in\left[a+\frac{2 n-1}{2} h, a+\frac{2 n-1+\beta(\gamma)}{2} h\right], \\
\frac{(b-x)^{6}}{6 !}-\frac{h}{2 \times 5 !} \gamma\left(a+\frac{2 n-1+\alpha(\beta(\gamma), \gamma)}{2} h-x\right)^{5} & \text { for } x \in\left[a+\frac{2 n-1+\beta(\gamma)}{2} h, a+\frac{2 n-1+\alpha(\beta(\gamma), \gamma)}{2} h\right], \\
\frac{(b-x)^{6}}{6 !} & \text { for } x \in\left[a+\frac{2 n-1+\alpha(\beta(\gamma), \gamma)}{2} h, b\right]
\end{aligned}\right.
$$

The kernel $K_{6}^{\mathcal{S}}(\gamma, x)$ is negative for $\gamma \in\left[\frac{1}{6}, \hat{\gamma}_{1}\right)$ and positive for $\gamma \in\left(\hat{\gamma}_{2}, \frac{5}{9}\right]$. After numerical calculation we conclude that $\hat{\gamma}_{1}>\frac{1}{4}, \hat{\gamma}_{2}<\sqrt{\frac{133}{1000}}$ (see Figure 2). 

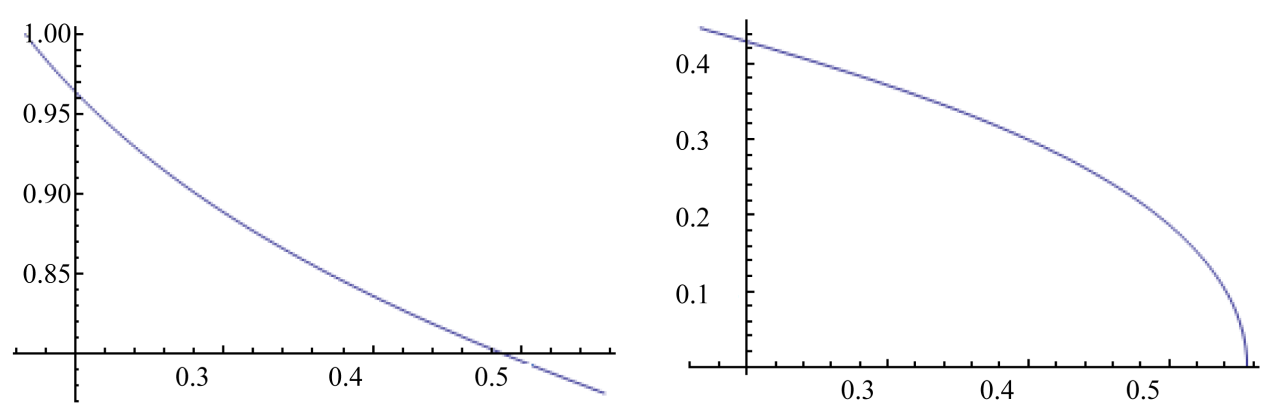

Figure 1. Graphs of $\alpha(\beta(\gamma), \gamma)$ and $\beta(\gamma)$.

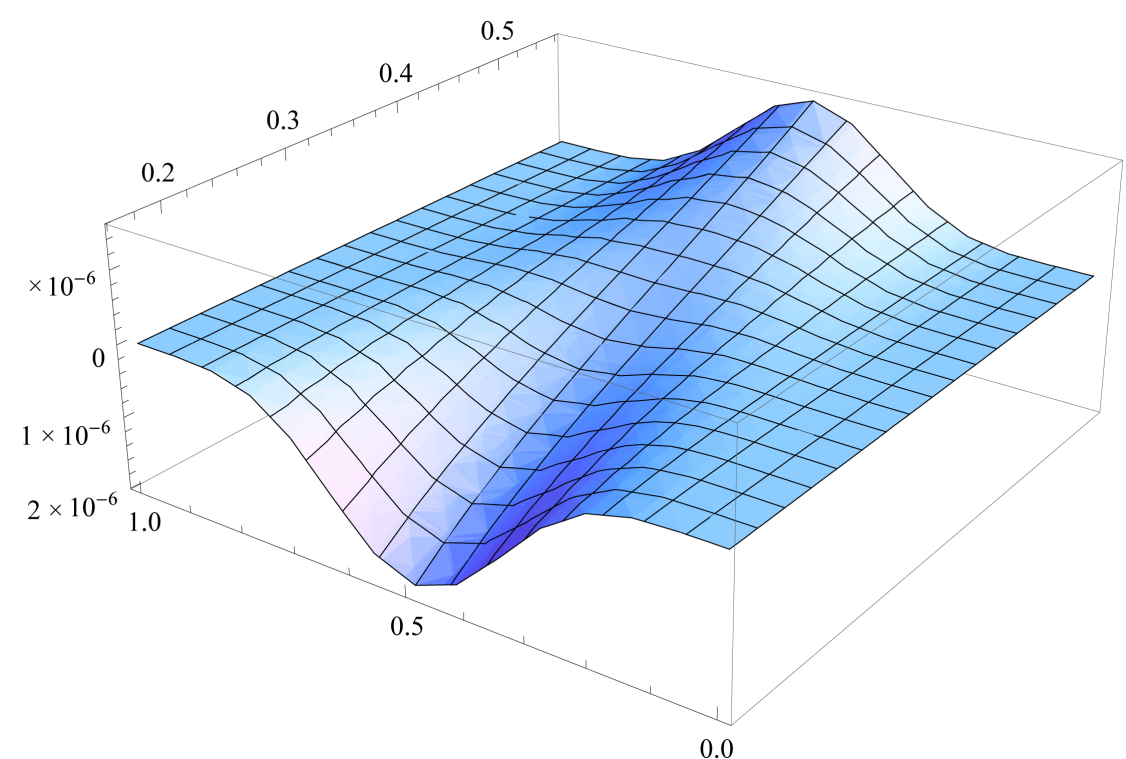

Figure 2. Graph of the kernel $K_{6}^{\mathcal{S}}(\gamma, x)$ for $x \in[b-h, b] \quad([a, b]=[-9,1], n=10)$.

The integral of the six order Peano kernel takes form

$$
c(\gamma)=\int_{a}^{b} K_{6}^{\mathcal{S}}(\gamma, x) \mathrm{d} x=\frac{b-a}{27216000} h^{6} \frac{(7-14 \gamma) \sqrt{5-5 \gamma}+10\left(\gamma^{\frac{3}{3}}-\sqrt{\gamma}\right)}{(\gamma-1) \sqrt{\gamma}}
$$

(see Figure 3).

From Peano theorem (see [5]) the error

$$
E^{\alpha(\beta(\gamma), \gamma), \beta(\gamma), \gamma}(f)=\frac{b-a}{27216000} h^{6} \frac{(1-14 \gamma) \sqrt{5-5 \gamma}+10\left(\gamma^{\frac{3}{2}}-\sqrt{\gamma}\right)}{(\gamma-1) \sqrt{\gamma}} f^{(6)}(\xi)=c(\gamma) f^{(6)}(\xi),
$$

for any function $f \in C^{6}([a, b])$ and $\gamma \in\left[\frac{1}{6}, \frac{1}{4}\right] \cup\left[\sqrt{\frac{133}{1000}}, \frac{5}{9}\right]$, where $\xi \in[a, b]$. Moreover, using Peano theorem we can prove the following:

Theorem 1. If $\gamma_{1} \in\left[\frac{1}{6}, \hat{\gamma}_{1}\right), \gamma_{2} \in\left(\hat{\gamma}_{2}, \frac{5}{9}\right]$, function $f \in C^{6}[a, b]$, and $f^{(6)}$ has constant sign on interval $[a, b]$, then 


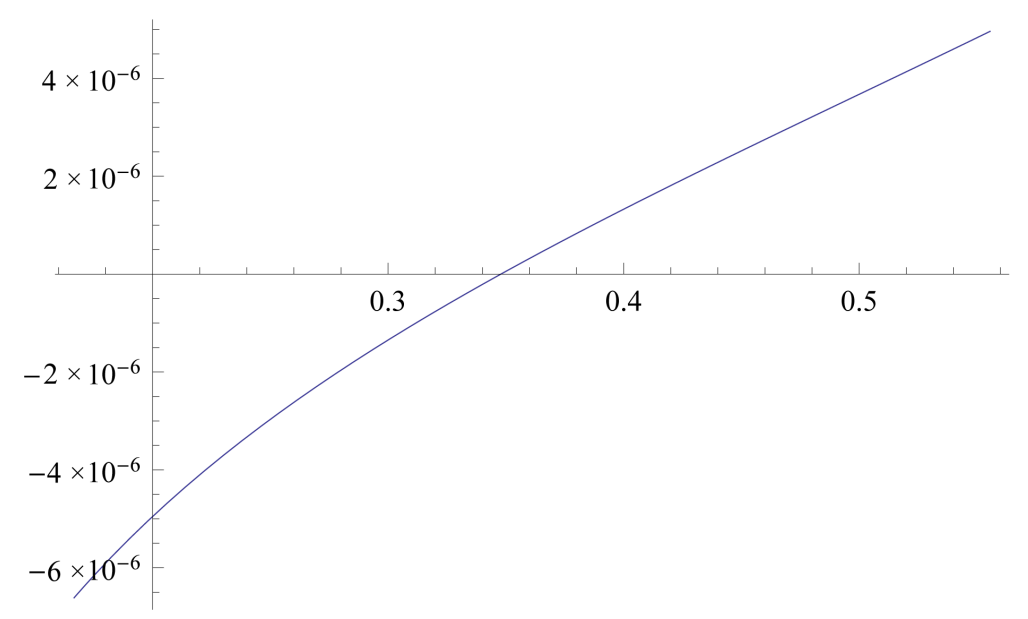

Figure 3. Graph of the function $c(\gamma)$.

$$
\mathcal{S}^{\alpha\left(\beta\left(\gamma_{2}\right), \gamma_{2}\right), \beta\left(\gamma_{2}\right), \gamma_{2}} \leq I(f) \leq \mathcal{S}^{\alpha\left(\beta\left(\gamma_{1}\right), \gamma_{1}\right), \beta\left(\gamma_{1}\right), \gamma_{1}}
$$

if $f^{(6)}$ is non-negative on interval $[a, b]$, and

$$
\mathcal{S}^{\alpha\left(\beta\left(\gamma_{1}\right), \gamma_{1}\right), \beta\left(\gamma_{1}\right), \gamma_{1}} \leq I(f) \leq \mathcal{S}^{\alpha\left(\beta\left(\gamma_{2}\right), \gamma_{2}\right), \beta\left(\gamma_{2}\right), \gamma_{2}}
$$

if $f^{(6)}$ is non-positive on interval $[a, b]$.

Proof. Assume that $f^{(6)} \geq 0$. From the formula (3), because of $c\left(\gamma_{1}\right)<0$ and $f^{(6)} \geq 0$, we have

$$
I(f)=\mathcal{S}^{\alpha\left(\beta\left(\gamma_{1}\right), \gamma_{1}\right), \beta\left(\gamma_{1}\right), \gamma_{1}}+c\left(\gamma_{1}\right) f^{(6)}\left(\xi_{1}\right) \leq \mathcal{S}^{\alpha\left(\beta\left(\gamma_{1}\right), \gamma_{1}\right), \beta\left(\gamma_{1}\right), \gamma_{1}} .
$$

Similarly

$$
I(f)=\mathcal{S}^{\alpha\left(\beta\left(\gamma_{2}\right), \gamma_{2}\right), \beta\left(\gamma_{2}\right), \gamma_{2}}+c\left(\gamma_{2}\right) f^{(6)}\left(\xi_{2}\right) \geq \mathcal{S}^{\alpha\left(\beta\left(\gamma_{2}\right), \gamma_{2}\right), \beta\left(\gamma_{2}\right), \gamma_{2}}
$$

because of $c\left(\gamma_{2}\right)>0$ and $f^{(6)} \geq 0$.

The function $\psi_{3}(\gamma):=(14 \gamma-7) \sqrt{5-5 \gamma}+10 \sqrt{\gamma}-10 \gamma^{\frac{3}{2}}$ has one root $\gamma_{0}=\frac{1}{36}(18-\sqrt{30})=\frac{49}{6(18+\sqrt{30})}=\frac{1}{2}-\frac{1}{6} \sqrt{\frac{5}{6}} \approx 0.3478548451$. Lets put $\beta_{0}=\beta\left(\gamma_{0}\right)=\sqrt{\frac{3}{7}-\frac{2}{7} \sqrt{\frac{6}{5}}} \approx 0.3399810436, \alpha_{0}=\alpha\left(\beta_{0}, \gamma_{0}\right)=\sqrt{\frac{3}{7}+\frac{2}{7} \sqrt{\frac{6}{5}}} \approx 0.8611363116$. The quadrature formula $\mathcal{S}^{\alpha_{0}, \beta_{0}, \gamma_{0}}$ is of the eight order and

$$
\begin{aligned}
& E^{\alpha_{0}, \beta_{0}, \gamma_{0}}\left(t^{i}\right)=0 \quad \text { for } i=1, \cdots, 7 \\
& E^{\alpha_{0}, \beta_{0}, \gamma_{0}}\left(t^{8}\right)=\frac{(b-a)^{9}}{44100 n^{8}}=\frac{h^{8}}{44100}(b-a) .
\end{aligned}
$$

The eight order Peano kernel $K_{8}^{\mathcal{S}}(x)=\frac{1}{7 !} \int_{x}^{b}(t-x)^{7} \mathrm{~d} t-\mathcal{S}^{\alpha_{0}, \beta_{0}, \gamma_{0}}(p(t))$ where $p(t)=\frac{(t-x)_{+}^{7}}{7 !}$. This kernel is a periodic function with period $h$ and on every interval $[a+i h, a+(i+1) h]$ symmetrical with respect to its midpoint. So us for $K_{8}^{\mathcal{S}}$, it is enough to define it on the interval $\left[b-\frac{h}{2}, b\right]$ : 


$$
K_{8}^{\mathcal{S}}(x)=\left\{\begin{aligned}
\frac{(b-x)^{8}}{8 !}-\frac{h}{2 \times 7 !}\left(\gamma_{0}\left(a+\frac{2 n-1+\alpha_{0}}{2} h-x\right)^{7}+\left(1-\gamma_{0}\right)\left(a+\frac{2 n-1+\beta_{0}}{2} h-x\right)^{7}\right) \\
\quad \text { for } x \in\left[a+\frac{2 n-1}{2} h, a+\frac{2 n-1+\beta_{0}}{2} h\right], \\
\quad \text { for } x \in\left[a+\frac{2 n-1+\beta_{0}}{2} h, a+\frac{2 n-1+\alpha_{0}}{2} h\right], \\
\text { for } x \in\left[a+\frac{2 n-1+\alpha_{0}}{2} h, b\right]
\end{aligned}\right.
$$

(see Figure 4).

This kernel $K_{8}^{\mathcal{S}}(x)$ is non-negative, moreover

$$
\int_{a}^{b} K_{8}^{\mathcal{S}}(x) \mathrm{d} x=2 n \int_{b-\frac{h}{2}}^{b} K_{8}^{\mathcal{S}}(x) \mathrm{d} x=\frac{(b-a)^{9}}{1778112000 n^{8}}=\frac{h^{8}}{1778112000}(b-a) .
$$

From the Peano theorem (see [5]) we obtain for any function $f \in C^{8}([a, b])$ the expression on the error

$$
E^{\alpha_{0}, \beta_{0}, \gamma_{0}}(f)=\frac{h^{8}}{1778112000}(b-a) f^{(8)}(\xi) \text {, }
$$

where $\xi \in[a, b]$.

\section{The Three-Parameter Family of Quadrature Formulas $\mathcal{P}^{\alpha, \beta, \gamma}$}

We consider the family of quadrature formulas of the form

$$
\mathcal{P}^{\alpha, \beta, \gamma}(f):=T_{n}(f)+\gamma G_{n}(f, \alpha)+(1-\gamma) G_{n}(f, \beta)
$$

where

$$
G_{n}(f, \lambda)=\frac{h}{24 \lambda}\left(-3\left(f_{0}+f_{n}\right)+4\left(f_{\lambda}+f_{n-\lambda}\right)-\left(f_{2 \lambda}+f_{n-2 \lambda}\right)\right),
$$

$f_{t}:=f(a+t h), h=\frac{b-a}{n}, T_{n}(f)=\frac{h}{2}\left(f_{0}+f_{n}\right)+h \sum_{i=1}^{n-1} f_{i}$ is the trapezoidal rule, and $\alpha, \beta, \gamma$ are para-

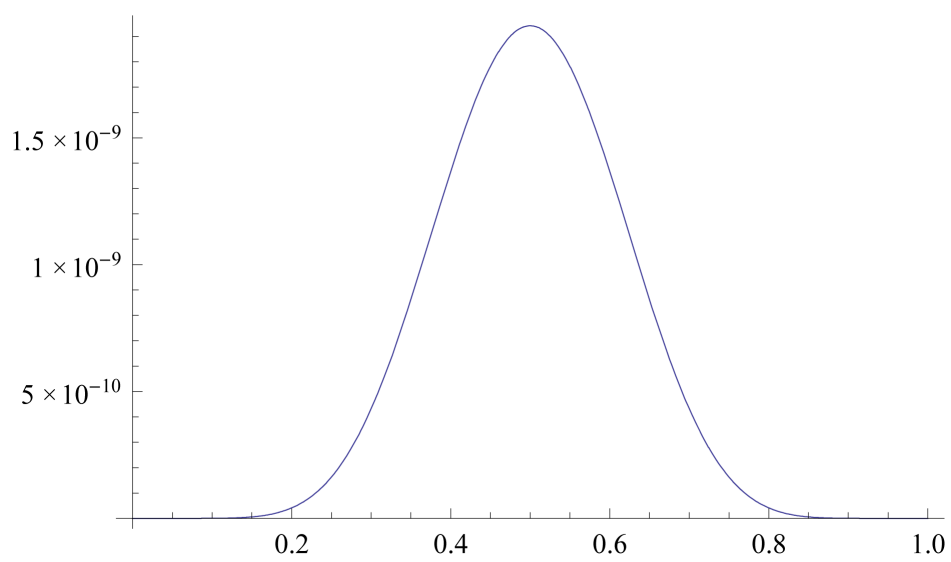

Figure 4. Graph of the fragment of the kernel $K_{8}^{\mathcal{S}}(x)$ for $x \in[b-h, b]$ $([a, b]=[-9,1], \quad n=10)$. 
meters. Particular cases $\gamma=1$ and $\gamma=0$ are investigated in the paper [2] and $\mathcal{P}^{\alpha, \beta, 1}(f)=Q_{n+5}^{\alpha}(f)$, $\mathcal{P}^{\alpha, \beta, 0}(f)=Q_{n+5}^{\beta}(f)$. We are proved that $Q_{n+5}^{\alpha_{n}}$ and $Q_{n+5}^{\beta_{n}}$ with

$$
\begin{aligned}
& \alpha_{n}=\frac{2}{9} n\left(1+2 \cos \left(\frac{\varphi_{n}+2 \pi}{3}\right)\right), \\
& \beta_{n}=\frac{2}{9} n\left(1+2 \cos \left(\frac{\varphi_{n}+4 \pi}{3}\right)\right),
\end{aligned}
$$

where $\varphi_{n} \in\left(0, \frac{\pi}{2}\right)$ and $\varphi_{n}=\arccos \left(1-\frac{243}{160 n^{2}}\right)$ (see Figure 5) are of the six order. If we define the error $E^{\alpha, \beta, \gamma}(f)=I(f)-\mathcal{P}^{\alpha, \beta, \gamma}(f)$ we can compute for the polynomials $t, t^{2}, \cdots$

$$
\begin{aligned}
& E^{\alpha_{n}, \beta_{n}, \gamma}\left(t^{k}\right)=0 \quad \text { for } k=1, \cdots, 5, \\
& E^{\alpha_{n}, \beta_{n}, \gamma}\left(t^{6}\right)=-\frac{(b-a)^{7}}{8266860 n^{6}}\left(-W_{n}+560 n^{4}\left(V_{n}+\sqrt{3}(2 \gamma-1) U_{n}\right)\right), \\
& E^{\alpha_{n}, \beta_{n}, \gamma}\left(t^{7}\right)=-\frac{(b-a)^{7}(a+b)}{2361960 n^{6}}\left(-W_{n}+560 n^{4}\left(V_{n}+\sqrt{3}(2 \gamma-1) U_{n}\right)\right),
\end{aligned}
$$

where

$$
\begin{aligned}
& W_{n}=81\left(560 n^{4}+63 n^{2}-2430\right), \\
& U_{n}=198 \sin \frac{1}{3} \varphi_{n}+105 \sin \frac{2}{3} \varphi_{n}-52 \sin \frac{4}{3} \varphi_{n}-40 \sin \frac{5}{3} \varphi_{n}, \\
& V_{n}=-198 \cos \frac{1}{3} \varphi_{n}+105 \cos \frac{2}{3} \varphi_{n}+52 \cos \frac{4}{3} \varphi_{n}-40 \cos \frac{5}{3} \varphi_{n} .
\end{aligned}
$$

So, for every $\gamma \in[0,1]$ the quadrature $\mathcal{P}^{\alpha_{n}, \beta_{n}, \gamma}$ is of the six order. Let

$$
\gamma_{n}=\frac{W_{n}+560 n^{4}\left(V_{n}+\sqrt{3} U_{n}\right)}{1120 \sqrt{3} n^{4} U_{n}} .
$$

With $\gamma=\gamma_{n}$ the range of quadrature formula increases. The quadrature $\mathcal{P}^{\alpha_{n}, \beta_{n}, \gamma_{n}}$ is of the eight order but the expression $E^{\alpha_{n}, \beta_{n}, \gamma_{n}}\left(t^{8}\right)$ takes a very complicated form.

The eight order Peano kernel $K_{8}^{\mathcal{P}}(x)=\frac{1}{7 !} \int_{x}^{b}(t-x)^{7} \mathrm{~d} t-\mathcal{P}^{\alpha_{n}, \beta_{n}, \gamma_{n}}(p(t))$ where $p(t)=\frac{(t-x)_{+}^{7}}{7 !}$. This kernel
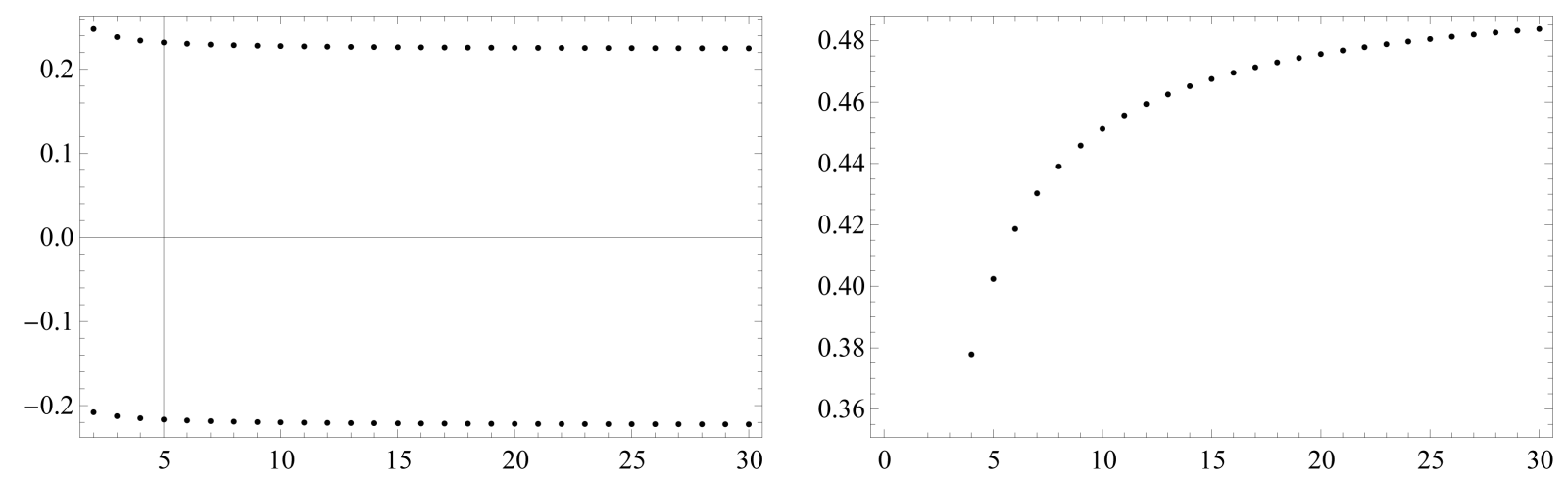

Figure 5. Graphs of the sequences $\alpha_{n}, \beta_{n}, \gamma_{n}$. 
is a symmetrical function respect to the point $\frac{a+b}{2}$, so it is enough to define it on the interval $\left[\frac{a+b}{2}, b\right]$ :

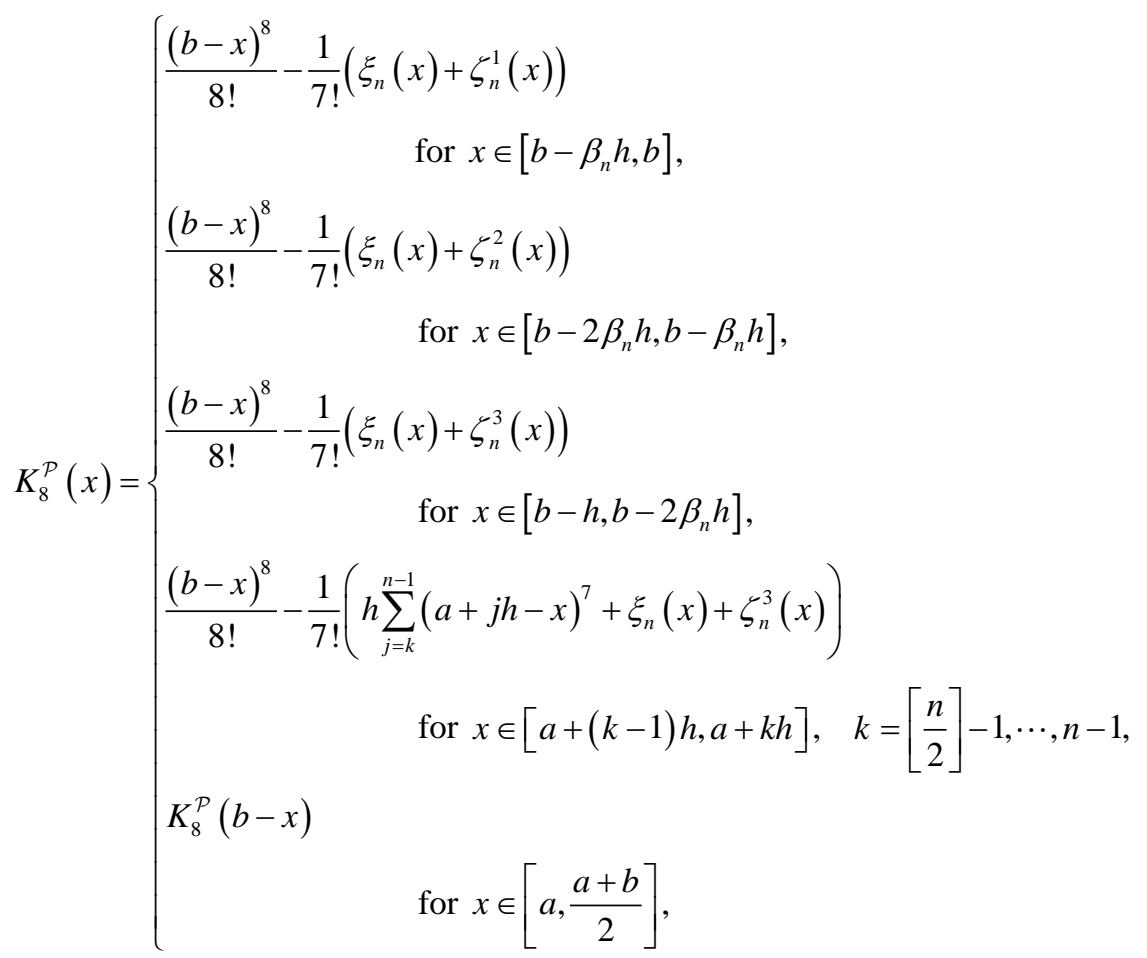

where

$$
\begin{aligned}
& \xi_{n}(x)=\frac{h}{2}(b-x)^{7}+\frac{\gamma_{n} h}{24 \alpha_{n}}\left(-3(b-x)^{7}+4\left(b-\alpha_{n} h-x\right)^{7}-\left(b-2 \alpha_{n} h-x\right)^{7}\right), \\
& \zeta_{n}^{1}(x)=\frac{\left(1-\gamma_{n}\right) h}{24 \beta_{n}}\left(-3(b-x)^{7}\right), \\
& \zeta_{n}^{2}(x)=\frac{\left(1-\gamma_{n}\right) h}{24 \beta_{n}}\left(-3(b-x)^{7}+4\left(b-\beta_{n} h-x\right)^{7}\right), \\
& \zeta_{n}^{3}(x)=\frac{\left(1-\gamma_{n}\right) h}{24 \beta_{n}}\left(-3(b-x)^{7}+4\left(b-\beta_{n} h-x\right)^{7}-\left(b-2 \beta_{n} h-x\right)^{7}\right),
\end{aligned}
$$

and $h=\frac{b-a}{n}$. On the Figure 6 we have graphs of the kernels $K_{8}^{\mathcal{P}}(x)$ for $n=4,5,6$. For any $n$ the kernel $K_{8}^{\mathcal{P}}(x)$ is non-positive, moreover the integral

$$
\begin{aligned}
C(n):= & \int_{a}^{b} K_{8}^{\mathcal{P}}(x) \mathrm{d} x \\
= & \frac{(b-a)^{9}}{928972800 m^{9}}\left(945\left(1-\gamma_{n}\right) \beta_{n}^{7}+m\left(-3720 \gamma_{n} \alpha_{n}^{6}-3720\left(1-\gamma_{n}\right) \beta_{n}^{6}+3\right)\right. \\
& +6300 m^{2}\left(\gamma_{n} \alpha_{n}^{5}+\left(1-\gamma_{n}\right) \beta_{n}^{5}\right)-20 m^{3}\left(294 \gamma_{n}^{4}+294\left(1-\gamma_{n}\right) \beta_{n}^{4}+1\right) \\
& \left.+3150 m^{4}\left(\gamma_{n} \alpha_{n}^{3}+\left(1-\gamma_{n}\right) \beta_{n}^{3}\right)+42 m^{5}\left(-20 \gamma_{n} \alpha_{n}^{2}-20\left(1-\gamma_{n}\right) \beta_{n}^{2}+1\right)\right)
\end{aligned}
$$

in the case $n=2 m$ and 


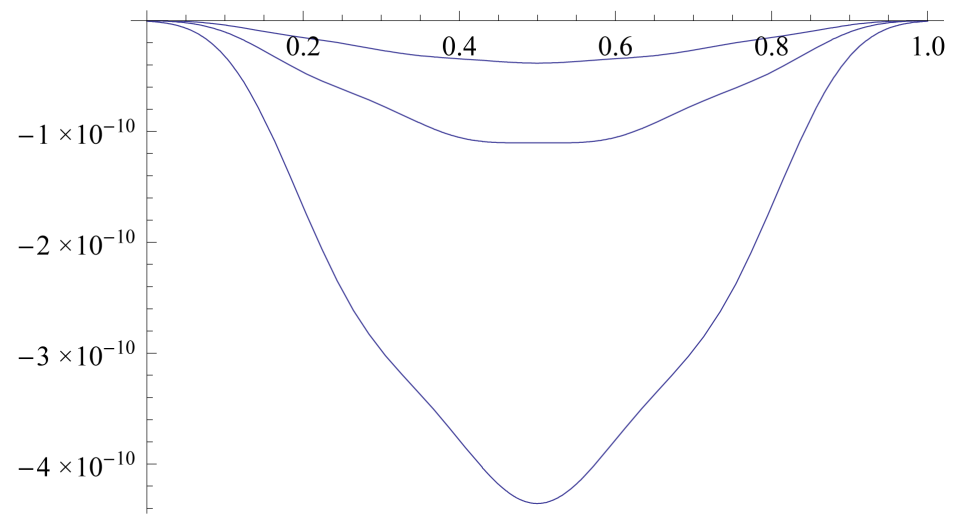

Figure 6. Graphs of the kernels $K_{8}^{\mathcal{P}}(x)$ for $n=4,5,6$.

$$
\begin{aligned}
C(n):= & \int_{a}^{b} K_{8}^{\mathcal{P}}(x) \mathrm{d} x \\
= & \frac{(b-a)^{9}}{3628800(2 m+1)^{9}}\left(-45\left(\gamma_{n}\left(616 \alpha_{n}^{2}-1190 \alpha_{n}^{3}+1176 \alpha_{n}^{4}-700 \alpha_{n}^{5}+248 \alpha_{n}^{6}\right)\right.\right. \\
& \left.+\left(1-\gamma_{n}\right)\left(616 \beta_{n}^{2}-1190 \beta_{n}^{3}+1176 \beta_{n}^{4}-700 \beta_{n}^{5}+248 \beta_{n}^{6}\right)-27\right) \\
& +12 m\left(\gamma_{n}\left(-5950 \alpha_{n}^{2}+9450 \alpha_{n}^{3}-7350 \alpha_{n}^{4}+3150 \alpha_{n}^{5}-620 \alpha_{n}^{6}\right)\right. \\
& \left.+\left(1-\gamma_{n}\right)\left(-5950 \beta_{n}^{2}+9450 \beta_{n}^{3}-7350 \beta_{n}^{4}+3150 \beta_{n}^{5}-620 \beta_{n}^{6}\right)+273\right) \\
& +180 m^{2}\left(\gamma_{n}\left(420 \alpha_{n}^{2}-525 \alpha_{n}^{3}+294 \alpha_{n}^{4}-70 \alpha_{n}^{5}\right)\right. \\
& \left.+\left(1-\gamma_{n}\right)\left(420 \beta_{n}^{2}-525 \beta_{n}^{3}+294 \beta_{n}^{4}-70 \beta_{n}^{5}\right)-20\right) \\
& +20 m^{3}\left(\gamma_{n}\left(-2100 \alpha_{n}^{2}+1890 \alpha_{n}^{3}-588 \alpha_{n}^{4}\right)\right. \\
& \left.+\left(1-\gamma_{n}\right)\left(-2100 \beta_{n}^{2}+1890 \beta_{n}^{3}-588 \beta_{n}^{4}\right)+103\right) \\
& -630 m^{4}\left(\gamma_{n}\left(20 \alpha_{n}^{2}-10 \alpha_{n}^{3}\right)+\left(1-\gamma_{n}\right)\left(20 \beta_{n}^{2}-10 \beta_{n}^{3}\right)-1\right) \\
& \left.+84 m^{5}\left(-20 \gamma_{n} \alpha_{n}^{2}-20\left(1-\gamma_{n}\right) \beta_{n}^{2}+1\right)\right)
\end{aligned}
$$

if $n=2 m+1$. From the Peano theorem (see [5]) we obtain for any function $f \in C^{8}([a, b])$ the expression on the error

$$
E^{\alpha_{n}, \beta_{n}, \gamma_{n}}(f)=C(n) f^{(8)}(\xi)
$$

where $\xi \in[a, b]$ and $C(n)<0$ for all $n$.

A Complex Quadrature Formula $\mathcal{P}_{m}^{\alpha, \beta, \gamma}(f)$

Let $m \in \mathbb{N}$, the step $k=\frac{b-a}{m}$ and the nodes $x_{j}=a+j k \quad(j=0,1, \cdots, m)$. The integral $I(f)$ can be written in the form $I(f)=\sum_{j=0}^{m-1} I_{j}(f)$, where $I_{j}(f)=\int_{x_{j}}^{x_{j+1}} f(x) \mathrm{d} x$. To each integral $I_{j}(f)$ we apply the quadrature (8):

$$
\mathcal{P}_{(j)}^{\alpha, \beta, \gamma}(f):=T_{j, n}(f)+\gamma G_{j, n}(f, \alpha)+(1-\gamma) G_{j, n}(f, \beta)
$$


where now $G_{j, n}(f, \lambda)=\frac{h}{24 \lambda}\left(-3\left(f_{j, 0}+f_{j, n}\right)+4\left(f_{j, \lambda}+f_{j, n-\lambda}\right)-\left(f_{j, 2 \lambda}+f_{j, n-2 \lambda}\right)\right)$,

$$
\begin{aligned}
T_{j, n}(f)=\frac{h}{2}\left(f_{j, 0}+f_{j, n}\right)+ & h \sum_{i=1}^{n-1} f_{j, i}, \quad f_{j, t}:=f\left(x_{j}+t h\right), \quad h=\frac{x_{j+1}-x_{j}}{n}=\frac{b-a}{m n} . \text { Next we define } \\
\mathcal{P}_{m}^{\alpha, \beta, \gamma}(f)= & \sum_{j=0}^{m-1} \mathcal{P}_{(j)}^{\alpha, \beta, \gamma}(f)=h \sum_{j=0}^{m-1 n-1} f_{i=1} f_{j, i}+\frac{h \gamma}{24 \alpha} \sum_{j=0}^{m-1}\left(4\left(f_{j, \alpha}+f_{j, n-\alpha}\right)-\left(f_{j, 2 \alpha}+f_{j, n-2 \alpha}\right)\right) \\
& +\frac{h(1-\gamma)}{24 \beta} \sum_{j=0}^{m-1}\left(4\left(f_{j, \beta}+f_{j, n-\beta}\right)-\left(f_{j, 2 \beta}+f_{j, n-2 \beta}\right)\right) \\
& +h\left(\frac{1}{2}-\frac{\gamma}{8 \alpha}-\frac{1-\gamma}{8 \beta}\right)\left(\left(f_{0,0}+f_{m, 0}\right)+2 \sum_{j=1}^{m-1} f_{j, 0}\right) .
\end{aligned}
$$

Obviously $\mathcal{P}_{1}^{\alpha, \beta, \gamma}(f)=\mathcal{P}^{\alpha, \beta, \gamma}(f)$. For every $m \in \mathbb{N}$, the quadrature formula $\mathcal{P}_{m}^{\alpha_{n}, \beta_{n}, \gamma}(f)$ is of the six order and $\mathcal{P}_{m}^{\alpha_{n}, \beta_{n}, \gamma_{n}}(f)$ is of the eight order. The Peano kernel for the quadrature formula $\mathcal{P}_{m}^{\alpha, \beta, \gamma}(f)$ is a periodic function with period $k$ and on every interval $\left[x_{j}, x_{j+1}\right]$ is symmetrical with respect to its midpoint. The quadrature formula (12) has $m(n+4)+1$ nodes.

Because of Peano kernels for quadrature formulas $\mathcal{S}^{\alpha_{0}, \beta_{0}, \gamma_{0}}, \mathcal{P}^{\alpha_{n}, \beta_{n}, \gamma_{n}}\left(\mathcal{P}_{m}^{\alpha_{n}, \beta_{n}, \gamma_{n}}\right)$ have different signs, we have the following theorem.

Theorem 2. If function $f \in C^{8}[a, b]$, and the derivative $f^{(8)}$ has constant sign on interval $[a, b]$, then

$$
\mathcal{S}^{\alpha_{0}, \beta_{0}, \gamma_{0}}(f) \leq I(f) \leq \mathcal{P}^{\alpha_{n}, \beta_{n}, \gamma_{n}}(f) \quad\left(\mathcal{S}^{\alpha_{0}, \beta_{0}, \gamma_{0}}(f) \leq I(f) \leq \mathcal{P}_{m}^{\alpha_{n}, \beta_{n}, \gamma_{n}}(f)\right)
$$

if $f^{(8)}$ is non-negative on the interval $[a, b]$, and

$$
\mathcal{S}^{\alpha_{0}, \beta_{0}, \gamma_{0}}(f) \geq I(f) \geq \mathcal{P}^{\alpha_{n}, \beta_{n}, \gamma_{n}}(f) \quad\left(\mathcal{S}^{\alpha_{0}, \beta_{0}, \gamma_{0}}(f) \geq I(f) \geq \mathcal{P}_{m}^{\alpha_{n}, \beta_{n}, \gamma_{n}}(f)\right)
$$

if $f^{(8)}$ is non-positive on the interval $[a, b]$.

Proof. Assume that $f^{(8)} \geq 0$. From the formula (7) we have

$$
I(f)=\mathcal{S}^{\alpha_{0}, \beta_{0}, \gamma_{0}}(f)+c\left(\gamma_{0}\right) f^{(8)}\left(\xi_{1}\right) \geq \mathcal{S}^{\alpha_{0}, \beta_{0}, \gamma_{0}}(f)
$$

because of $c\left(\gamma_{0}\right)>0$ and $f^{(8)} \geq 0$. Similarly from the formula (10):

$$
I(f)=\mathcal{P}^{\alpha_{n}, \beta_{n}, \gamma_{n}}(f)+C(n) f^{(8)}\left(\xi_{2}\right) \leq \mathcal{P}^{\alpha_{n}, \beta_{n}, \gamma_{n}}(f)
$$

because of $C(n)<0$ and $f^{(8)} \geq 0$.

\section{The Three-Parameter Family of Quadrature Formulas $\mathcal{R}^{\alpha, \beta, \gamma}$}

We consider the family of quadrature formulas of the form

$$
\mathcal{R}^{\alpha, \beta, \gamma}(f):=M_{n}(f)+\gamma H_{n}(f, \alpha)+(1-\gamma) H_{n}(f, \beta)
$$

where

$$
H_{n}(f, \lambda)=\frac{h}{24 \lambda}\left(2\left(f_{\frac{1}{2} \lambda}+f_{n-\frac{1}{2} \lambda}\right)-3\left(f_{\frac{3}{2} \lambda}+f_{n-\frac{3}{2} \lambda}\right)+\left(f_{\frac{5}{2} \lambda}+f_{n-\frac{5}{2} \lambda}\right)\right),
$$

$f_{t}:=f(a+t h), h=\frac{b-a}{n}, M_{n}(f)=h \sum_{i=0}^{n-1} f_{i+\frac{1}{2}}$ is the midpoint rule, and $\alpha, \beta, \gamma$ are parameters. Particular cases $\gamma=1$ and $\gamma=0$ are investigated in the paper [3] and $\mathcal{R}^{\alpha, \beta, 1}(f)=L_{n+6}^{\alpha}(f)$, $\mathcal{R}^{\alpha, \beta, 0}(f)=L_{n+6}^{\beta}(f)$. We are proved that $L_{n+6}^{\tilde{\alpha}_{n}}$ and $L_{n+6}^{\tilde{\beta}_{n}}$ with

$$
\tilde{\alpha}_{n}=\frac{23}{144} n\left(1+2 \cos \left(\frac{\tilde{\varphi}_{n}+2 \pi}{3}\right)\right) \text {, }
$$




$$
\tilde{\beta}_{n}=\frac{23}{144} n\left(1+2 \cos \left(\frac{\tilde{\varphi}_{n}+4 \pi}{3}\right)\right),
$$

where

$$
\tilde{\varphi}_{n} \in\left(0, \frac{\pi}{2}\right) \quad \text { and } \quad \tilde{\varphi}_{n}=\arccos \left(1-\frac{108864}{60835 n^{2}}\right)
$$

are of the six order. If we define the error $E^{\alpha, \beta, \gamma}(f)=I(f)-\mathcal{R}^{\alpha, \beta, \gamma}(f)$ we can compute for the polynomials $t, t^{2}, \cdots$

$$
\begin{aligned}
& E^{\tilde{\alpha}_{n}, \tilde{\beta}_{n}, \gamma}\left(t^{k}\right)=0 \quad \text { for } k=1, \cdots, 5, \\
& E^{\tilde{\alpha}_{n}, \tilde{\beta}_{n}, \gamma}\left(t^{6}\right)=-\frac{(b-a)^{7}}{8668430991360 n^{6}}\left(\tilde{W}_{n}+425845 n^{4}\left(\tilde{V}_{n}-\sqrt{3}(2 \gamma-1) \tilde{U}_{n}\right)\right)
\end{aligned}
$$

where

$$
\begin{aligned}
& \tilde{U}_{n}=183582 \sin \frac{1}{3} \tilde{\varphi}_{n}+126870 \sin \frac{2}{3} \tilde{\varphi}_{n}-63043 \sin \frac{4}{3} \tilde{\varphi}_{n}-37030 \sin \frac{5}{3} \tilde{\varphi}_{n}, \\
& \tilde{V}_{n}=-183582 \cos \frac{1}{3} \tilde{\varphi}_{n}+126870 \cos \frac{2}{3} \tilde{\varphi}_{n}+63043 \cos \frac{4}{3} \tilde{\varphi}_{n}-37030 \cos \frac{5}{3} \tilde{\varphi}_{n}, \\
& \tilde{W}_{n}=81\left(-2468413440+425279232 n^{2}+161395255 n^{4}\right) .
\end{aligned}
$$

So, for every $\gamma \in[0,1]$ the quadrature $\mathcal{R}^{\tilde{\alpha}_{n}, \tilde{\beta}_{n}, \gamma}$ is of the six order. Let

$$
\tilde{\gamma}_{n}=\frac{\tilde{W}_{n}+42584 n^{4}\left(\tilde{V}_{n}+\sqrt{3} \tilde{U}_{n}\right)}{85168 \sqrt{3} n^{4} \tilde{U}_{n}}
$$

(see Figure 7).

With $\gamma=\tilde{\gamma}_{n}$ the range of quadrature formula increases. The quadrature $\mathcal{R}^{\tilde{\alpha}_{n}, \tilde{\beta}_{n}, \tilde{\gamma}_{n}}$ is of the eight order but the expression $E^{\tilde{\alpha}_{n}, \tilde{\beta}_{n}, \tilde{\gamma}_{n}}\left(t^{8}\right)$ takes a very complicated form.

The eight order Peano kernel $K_{8}^{\mathcal{R}}(x)=\frac{1}{7 !} \int_{x}^{b}(t-x)^{7} \mathrm{~d} t-\mathcal{R}^{\tilde{\alpha}_{n}, \tilde{\beta}_{n}, \tilde{\gamma}_{n}}(p(t))$ where $p(t)=\frac{(t-x)_{+}^{7}}{7 !}$. This kernel is a symmetrical function respect to the point $\frac{a+b}{2}$, so it is enough to define it on the interval $\left[\frac{a+b}{2}, b\right]$ :
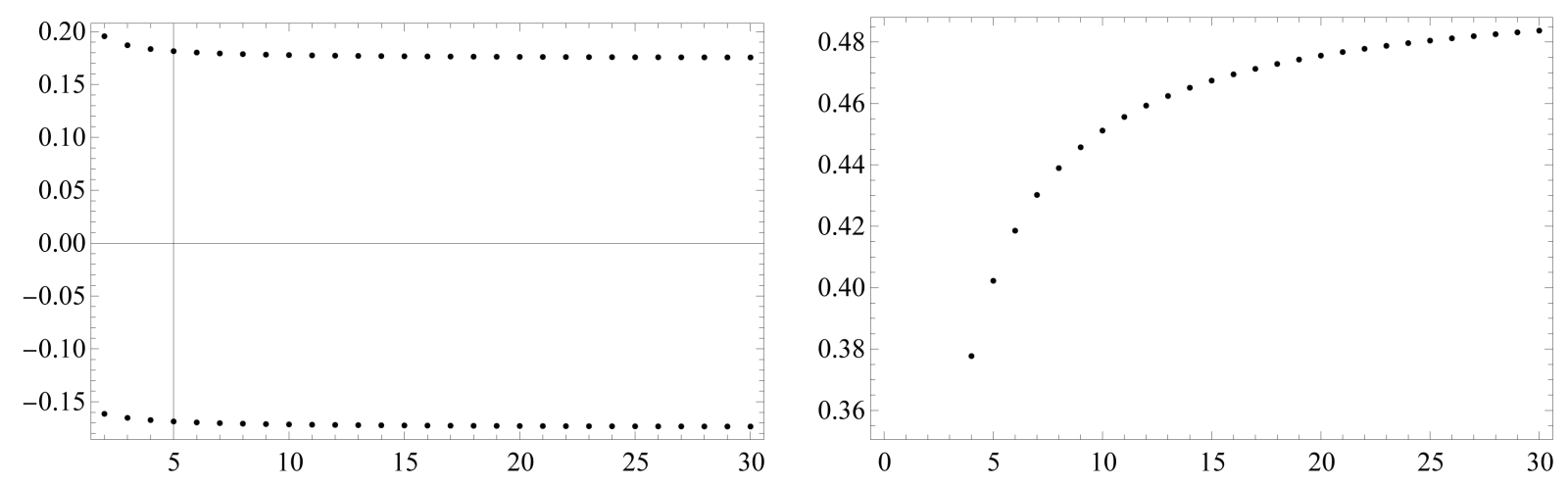

Figure 7. Graphs of the sequences $\tilde{\alpha}_{n}, \tilde{\beta}_{n}, \tilde{\gamma}_{n}$. 


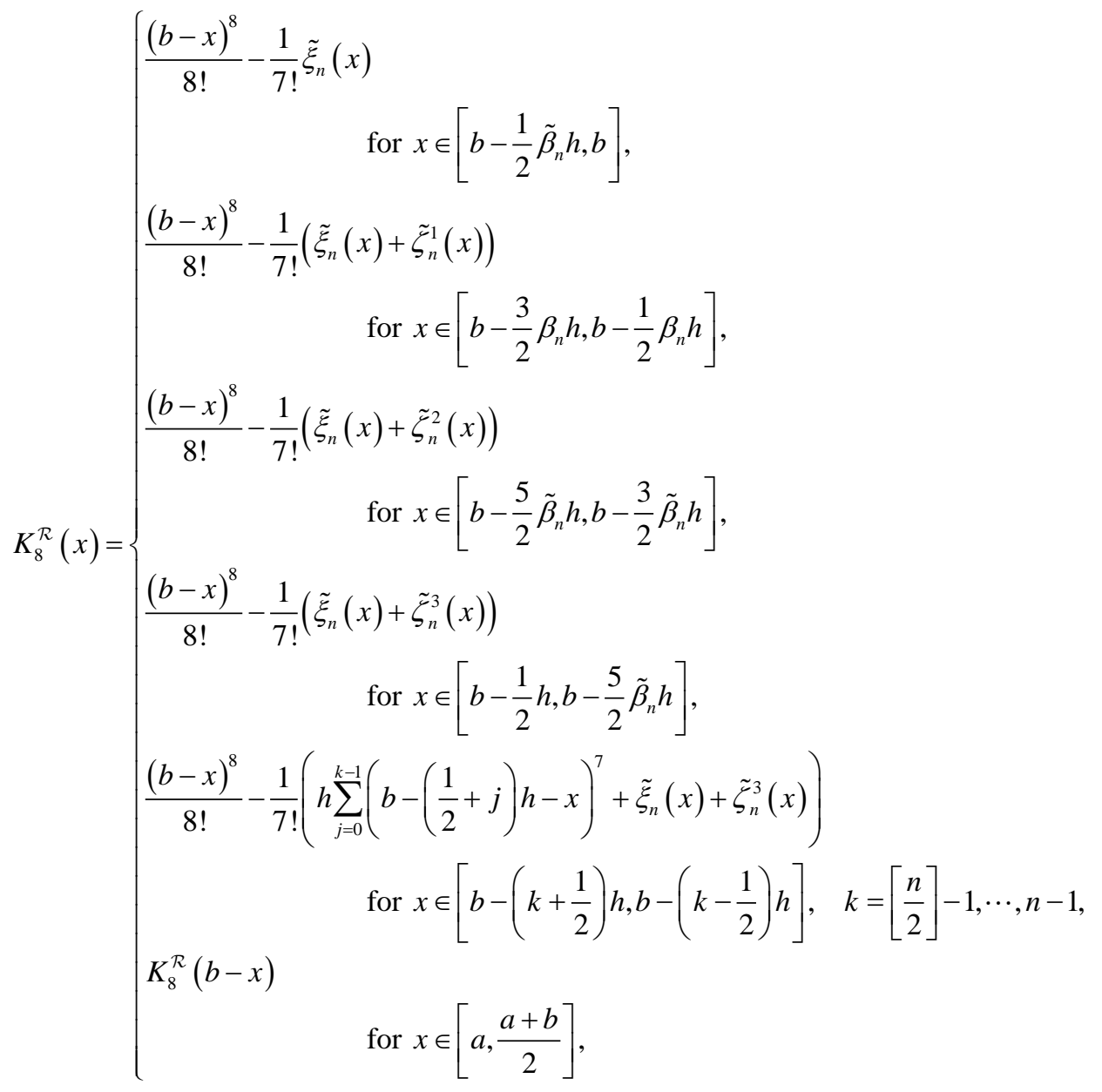

where

$$
\begin{aligned}
& \tilde{\xi}_{n}(x)=\frac{\tilde{\gamma}_{n} h}{24 \tilde{\alpha}_{n}}\left(2\left(b-\frac{1}{2} \tilde{\alpha}_{n} h-x\right)^{7}-3\left(b-\frac{3}{2} \tilde{\alpha}_{n} h-x\right)^{7}+\left(b-\frac{5}{2} \tilde{\alpha}_{n} h-x\right)^{7}\right), \\
& \tilde{\zeta}_{n}^{1}(x)=\frac{\left(1-\tilde{\gamma}_{n}\right) h}{12 \tilde{\beta}_{n}}\left(b-\frac{1}{2} \tilde{\beta}_{n} h-x\right)^{7}, \\
& \tilde{\zeta}_{n}^{2}(x)=\frac{\left(1-\tilde{\gamma}_{n}\right) h}{24 \tilde{\beta}_{n}}\left(2\left(b-\frac{1}{2} \tilde{\beta}_{n} h-x\right)^{7}-3\left(b-\frac{3}{2} \tilde{\beta}_{n} h-x\right)^{7}\right), \\
& \tilde{\zeta}_{n}^{3}(x)=\frac{\left(1-\tilde{\gamma}_{n}\right) h}{24 \tilde{\beta}_{n}}\left(2\left(b-\frac{1}{2} \tilde{\beta}_{n} h-x\right)^{7}-3\left(b-\frac{3}{2} \tilde{\beta}_{n} h-x\right)^{7}+\left(b-\frac{5}{2} \tilde{\beta}_{n} h-x\right)^{7}\right),
\end{aligned}
$$

and $h=\frac{b-a}{n}$. On the Figure 8 we have graphs of the kernels $K_{8}^{\mathcal{R}}(x)$ for $n=4,5,6$. For any $n$ the kernel $K_{8}^{\mathcal{R}}(x)$ is non-negative, moreover the integral

$$
\tilde{C}(n):=\int_{a}^{b} K_{8}^{\mathcal{R}}(x) \mathrm{d} x=-\frac{(b-a)^{9}}{464486400 n^{9}} \sum_{i=0}^{3} v_{i}\left(\tilde{\alpha}_{n}, \tilde{\beta}_{n}, \tilde{\gamma}_{n}\right) n^{i},
$$

where 


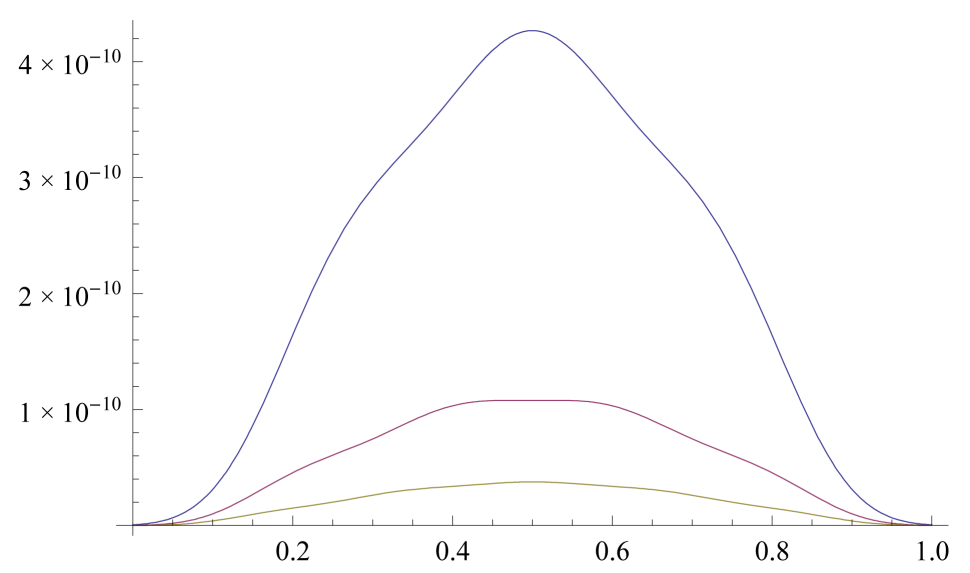

Figure 8. Graphs of the kernels $K_{8}^{\mathcal{R}}(x)$ for $n=4,5,6$.

$$
\begin{aligned}
& v_{0}(\alpha, \beta, \gamma)=20160\left(5 \alpha^{3}\left(1+14 \alpha^{2}\right) \gamma+\beta^{3}\left(5+70 \beta^{2}+69 \beta^{4}\right)(1-\gamma)\right) \\
& v_{1}(\alpha, \beta, \gamma)=-3\left(3+\mu_{1}(\alpha) \gamma+\mu_{1}(\beta)(1-\gamma)\right) \\
& v_{2}(\alpha, \beta, \gamma)=120\left(-18+\mu_{2}(\alpha) \gamma+\mu_{2}(\beta)(1-\gamma)\right) \\
& v_{3}(\alpha, \beta, \gamma)=80\left(116+\mu_{3}(\alpha) \gamma+\mu_{3}(\beta)(1-\gamma)\right) \\
& v_{4}(\alpha, \beta, \gamma)=1680\left(-7+\mu_{4}(\alpha) \gamma+\mu_{4}(\beta)(1-\gamma)\right) \\
& v_{5}(\alpha, \beta, \gamma)=672\left(7+\mu_{5}(\alpha) \gamma+\mu_{5}(\beta)(1-\gamma)\right)
\end{aligned}
$$

and

$$
\begin{aligned}
& \mu_{1}(\lambda)=20 \lambda^{2}(805+\lambda(6720+\lambda(25179+\lambda(47040+35783 \lambda)))) \\
& \mu_{2}(\lambda)=7 \lambda^{2}(230+3 \lambda(480+\lambda(1199+1120 \lambda))), \\
& \mu_{3}(\lambda)=-21 \lambda^{2}(230+\lambda(960+1199 \lambda)), \\
& \mu_{4}(\lambda)=10 \lambda^{2}(23+48 \lambda), \\
& \mu_{5}(\lambda)=-230 \lambda^{2} .
\end{aligned}
$$

From the Peano theorem (see [5]) we obtain for any function $f \in C^{8}([a, b])$ the expression on the error

$$
E^{\tilde{\alpha}_{n}, \tilde{\beta}_{n}, \tilde{\gamma}_{n}}(f)=\tilde{C}(n) f^{(8)}(\xi),
$$

where $\xi \in[a, b]$ and $\tilde{C}(n)>0$ for all $n$.

Theorem 3. If function $f \in C^{8}[a, b]$, and the derivative $f^{(8)}$ has constant sign on interval $[a, b]$, then

$$
\mathcal{R}^{\tilde{\alpha}_{n}, \tilde{\beta}_{n}, \tilde{\gamma}_{n}}(f) \leq I(f) \leq \mathcal{P}^{\alpha_{n}, \beta_{n}, \gamma_{n}}(f)
$$

if $f^{(8)}$ is non-negative on the interval $[a, b]$, and

$$
\mathcal{R}^{\tilde{\alpha}_{n}, \tilde{\beta}_{n}, \tilde{\gamma}_{n}}(f) \geq I(f) \geq \mathcal{P}^{\alpha_{n}, \beta_{n}, \gamma_{n}}(f)
$$

if $f^{(8)}$ is non-positive on the interval $[a, b]$.

Proof. Assume that $f^{(8)} \geq 0$. From the formulas (10) and (18):

$$
I(f)=\mathcal{R}^{\tilde{\alpha}_{n}, \tilde{\beta}_{n}, \tilde{\gamma}_{n}}(f)+\tilde{C}(n) f^{(8)}\left(\xi_{1}\right) \geq \mathcal{R}^{\tilde{\alpha}_{n}, \tilde{\beta}_{n}, \tilde{\gamma}_{n}}(f)
$$


because of $\tilde{C}(n)>0$ and $f^{(8)} \geq 0$ and

$$
I(f)=\mathcal{P}^{\alpha_{n}, \beta_{n}, \gamma_{n}}(f)+C(n) f^{(8)}\left(\xi_{2}\right) \leq \mathcal{P}^{\alpha_{n}, \beta_{n}, \gamma_{n}}(f)
$$

because of $C(n)<0$ and $f^{(8)} \geq 0$.

\section{Series Estimation}

The sum of a series

$$
s:=\sum_{n=0}^{\infty} a_{n}
$$

can be approximated by a finite sum $\sum_{n=0}^{N} a_{n}$. The error of this estimation can be represented as the sum of the series $\sum_{n=N+1}^{\infty} a_{n}$.

Therefore, if we have a method of estimating the sum of an infinite series, then this method will enable us to estimate the error of the $N$-term approximation. One way to estimate the sum of the series is to take into consideration the fact that a series can be viewed as an integral over an infinite domain

$$
I(f)=\int_{1}^{\infty} f(x) \mathrm{d} x
$$

for some function $f:[1, \infty) \rightarrow \mathbb{R}$ for which $f\left(n+\frac{1}{2}\right)=a_{n}$ for all $n$. Therefore, if for a given series, we know an explicitly integrable function $f(x)$ with this property, then we can take the value $I(f)$ of the integral as an estimate for $s$.

Theorem 4. We assume that the function $f$ is such that

1) $f$ is either positive and decreasing, or negative and increasing.

2) $\int_{1}^{\infty} f(x) \mathrm{d} x$ is convergent.

3) $f \in C^{8}([0, \infty))$.

4) $f^{(8)}$ is either positive or negative on $[0, \infty)$.

5) $f(j)=a_{j}$.

6) $s:=\sum_{n=0}^{\infty} a_{n}$.

Under this assumptions, if $f^{(8)}>0$ then

$$
\sum_{j=0}^{m-1} a_{j}+\frac{1}{2} a_{m}+\int_{m}^{\infty} f(x) \mathrm{d} x+P_{m}(f)<s<\sum_{j=0}^{m-1} a_{j}+\int_{m-\frac{1}{2}}^{\infty} f(x) \mathrm{d} x+\tilde{P}_{m}(f),
$$

where

$$
\begin{aligned}
P_{m}(f):=-\frac{\sqrt{5}}{24} & \left(4\left(f\left(m+\frac{\sqrt{5}}{10}\right)-f\left(m-\frac{\sqrt{5}}{10}\right)\right)-\left(f\left(m+\frac{\sqrt{5}}{5}\right)-f\left(m-\frac{\sqrt{5}}{5}\right)\right)\right), \\
\tilde{P}_{m}(f):= & \frac{1}{48} \sqrt{\frac{230}{7}}\left(2\left(f\left(m-\frac{1}{2}+\frac{1}{2} \sqrt{\frac{7}{230}}\right)-f\left(m-\frac{1}{2}-\frac{1}{2} \sqrt{\frac{7}{230}}\right)\right)\right. \\
& -3\left(f\left(m-\frac{1}{2}+\frac{3}{2} \sqrt{\frac{7}{230}}\right)-f\left(m-\frac{1}{2}-\frac{3}{2} \sqrt{\frac{7}{230}}\right)\right) \\
& \left.+\left(f\left(m-\frac{1}{2}+\frac{5}{2} \sqrt{\frac{7}{230}}\right)-f\left(m-\frac{1}{2}-\frac{5}{2} \sqrt{\frac{7}{230}}\right)\right)\right) .
\end{aligned}
$$

If $f^{(8)}>0$, then we get a similar inequality, but with the right-hand side instead of the left-hand side, and vice versa.

Proof. First, from the inequalities (19) we have: 


$$
I(f) \leq \mathcal{P}^{\alpha_{n}, \beta_{n}, \gamma_{n}}(f)=T_{n}(f)+\gamma_{n} G_{n}\left(f, \alpha_{n}\right)+\left(1-\gamma_{n}\right) G_{n}\left(f, \beta_{n}\right) .
$$

We can rewrite this inequality in an equivalent form:

$$
T_{n}(f) \geq I(f)-\gamma_{n} G_{n}\left(f, \alpha_{n}\right)-\left(1-\gamma_{n}\right) G_{n}\left(f, \beta_{n}\right) .
$$

In this inequality we put: $a=m,(m>2), h=1, n \geq 4$ so

$$
\begin{aligned}
T_{n}(f)= & \frac{1}{2}(f(m)+(m+n))+\sum_{i=1}^{n-1} f(m+i)=\frac{1}{2} a_{m}+\frac{1}{2} a_{m+n}+\sum_{j=m+1}^{m+n-1} a_{j}, \\
G_{n}(f, \lambda)= & \frac{1}{24 \lambda}(-3(f(m)+f(m+n))+4(f(m+\lambda)+f(m+n-\lambda)) \\
& -(f(m+2 \lambda)+f(m+n-2 \lambda))) .
\end{aligned}
$$

Because of

$$
\lim _{k \rightarrow \infty} a_{k}=0, \lim _{t \rightarrow \infty} f(t)=0, \lim _{n \rightarrow \infty} \alpha_{n}=-\frac{\sqrt{5}}{10}, \lim _{n \rightarrow \infty} \beta_{n}=\frac{\sqrt{5}}{10}, \lim _{n \rightarrow \infty} \gamma_{n}=\frac{1}{2}
$$

than passing with $n$ to $\infty$ in the inequality (24) we obtain

$$
\frac{1}{2} a_{m}+\sum_{j=m+1}^{\infty} a_{j} \geq \int_{m}^{\infty} f(x) \mathrm{d} x+P_{m}(f) .
$$

We complete the first part of the proof by adding the term $\frac{1}{2} a_{m}+\sum_{j=0}^{m-1} a_{j}$ to the both sides of this inequality. Let $\tilde{f}(x):=f\left(x-\frac{1}{2}\right)$. From the inequalities (19) we have:

$$
I(\tilde{f}) \geq \mathcal{R}^{\tilde{\alpha}_{n}, \tilde{\beta}_{n}, \tilde{\gamma}_{n}}(\tilde{f})=M_{n}(\tilde{f})+\tilde{\gamma}_{n} H_{n}\left(\tilde{f}, \tilde{\alpha}_{n}\right)+\left(1-\tilde{\gamma}_{n}\right) H_{n}\left(\tilde{f}, \tilde{\beta}_{n}\right) .
$$

We rewrite this inequality in an equivalent form:

$$
M_{n}(\tilde{f}) \leq I(\tilde{f})-\tilde{\gamma}_{n} H_{n}\left(\tilde{f}, \tilde{\alpha}_{n}\right)-\left(1-\tilde{\gamma}_{n}\right) H_{n}\left(\tilde{f}, \tilde{\beta}_{n}\right)
$$

and put: $a=m,(m>2), h=1, n \geq 4$. Passing with $n$ to $\infty$ we obtain

$$
\sum_{j=m}^{\infty} a_{j} \leq \int_{m-\frac{1}{2}}^{\infty} f(x) \mathrm{d} x+\tilde{P}_{m}(f)
$$

because of

$$
\lim _{k \rightarrow \infty} a_{k}=0, \lim _{t \rightarrow \infty} f(t)=0, \lim _{n \rightarrow \infty} \tilde{\alpha}_{n}=-\sqrt{\frac{7}{230}}, \lim _{n \rightarrow \infty} \tilde{\beta}_{n}=\sqrt{\frac{7}{230}}, \lim _{n \rightarrow \infty} \tilde{\gamma}_{n}=\frac{1}{2} .
$$

We complete the proof by adding the term $\sum_{j=0}^{m-1} a_{j}$ to the both sides of the inequality (25).

\section{Acknowledgements}

We thank the editor and the referee for their comments.

\section{References}

[1] Bożek, B., Solak, W. and Szydełko, Z. (2008) A Note on a Family of Quadrature Formulas and Some Applications. Opuscula Mathematica, 28, 109-121.

[2] Bożek, B., Solak, W. and Szydełko, Z. (2009) A Note of Some Quadrature Rules with Gregory End Corrections. Opuscula Mathematica, 29, 117-129.

[3] Bożek, B., Solak, W. and Szydełko, Z. (2012) On Some Quadrature Rules with Laplace End Corrections. Central Eu- 
ropean Journal of Mathematics, 10, 1172-1184. http://dx.doi.org/10.2478/s11533-012-0034-6

[4] de Villiers, J.M. (1993) A Nodal Spline Interpolant for the Gregory Rule of Even Order. Numerische Mathematik, 66, 123-137. http://dx.doi.org/10.1007/BF01385690

[5] Kincaid, D. and Cheney, W. (2002) Numerical Analysis, Mathematics of Scientific Computing. 3rd Edition, The University of Texas at Austin, Brooks/Cole-Thomson Learning. 